\title{
Pratiques
}

Linguistique, littérature, didactique

181-182| 2019

Le récit en questions

\section{Des récits et des élèves}

Stories and Pupils

\section{Yves Reuter}

\section{(2) OpenEdition \\ Journals}

Édition électronique

URL : http://journals.openedition.org/pratiques/5971

DOI : 10.4000/pratiques.5971

ISSN : 2425-2042

\section{Éditeur}

Centre de recherche sur les médiations (CREM)

\section{Référence électronique}

Yves Reuter, "Des récits et des élèves », Pratiques [En ligne], 181-182 | 2019, mis en ligne le 30 juin 2019, consulté le 20 juillet 2019. URL : http://journals.openedition.org/pratiques/5971 ; DOI : 10.4000/ pratiques. 5971

Ce document a été généré automatiquement le 20 juillet 2019.

(c) Tous droits réservés 


\title{
Des récits et des élèves
}

\author{
Stories and Pupils
}

\section{Yves Reuter}

1 Je me situe dans un cadre didactique "autonomiste ", c'est-à-dire dans une conception qui ne considère pas les didactiques comme des sous-disciplines ${ }^{1}$ de telle ou telle discipline universitaire, plus ancienne ou plus légitime. J'ai ainsi proposé de définir les didactiques de la manière suivante (Reuter, 2013, p. 65): «On pourrait définir, en première approche, les didactiques comme les disciplines de recherche qui analysent les contenus (savoirs, savoir faire...) en tant qu'ils sont objets d'enseignement et d'apprentissages, référés/référables à des matières scolaires. »

2 Cela signifie que les didactiques, comme toute discipline de recherche, produisent des questions qui leur sont propres, des concepts spécifiques, des méthodes ajustées ${ }^{2}$. Je vais donc tenter d'expliciter cela à partir de quelques questions qui concernent les fonctions des récits dans les différentes disciplines scolaires, la prise en compte des contenus, les méthodes d'analyse, le statut didactique des types de récits et la position d'apprentissage, en m'appuyant sur des recherches que j'ai pu mener, seul, avec des collègues ou avec des étudiants. Cela ne signifie nullement que je tienne les divers travaux qui contribuent à renouveler les études sur les récits ${ }^{3}$ comme négligeables ou non pertinents, cela signifie simplement qu'ils traitent, en fonction du cadre au sein duquel ils se situent, d'autres questions qui leur sont propres.

\section{Fonctions des récits dans les disciplines}

Dans la mesure où les récits sont présents dans la totalité des disciplines scolaires, les premières questions que je souhaite traiter ont trait aux fonctions des récits au sein des différentes disciplines. Cela fait écho à la proposition de J.-P. Astolfi (2008, p. 217) : " Aucune discipline n'a le monopole d'un contenu d'enseignement (puisqu'on n'apprend pas à lire qu'en français, ni à calculer qu'en mathématiques), mais chaque contenu a besoin d'une discipline de référence qui prenne en charge sa structuration. » 


\section{Quelles fonctions des récits dans les différentes disciplines scolaires?}

4 J'avais, dans un précédent numéro de Pratiques, tenté de construire une typologie des fonctions des récits dans les différentes disciplines scolaires ${ }^{4}$ (Reuter, 2007a). Je vais dans un premier temps revenir sur cette typologie pour lui faire subir quelques modifications, sachant que certaines fonctions sont très proches et peuvent être mises en œuvre en même temps.

1. Fonction d'objet d'enseignement : le récit en tant que tel est objet explicite d'enseignement.

2. Fonction d'objet d'apprentissages: le récit est objet d'apprentissages (mais il n'est pas forcément enseigné explicitement ${ }^{5}$ ).

3. Fonction d'objet d'évaluation: le récit est objet d'évaluation (directe ou indirecte, qu'il ait été enseigné explicitement ou implicitement).

4. Fonction structurante de la discipline : en lien avec la chronologie, le récit sert à établir des relations, à organiser les contenus au sein de la discipline (à la différence d'une organisation thématique, voir l'histoire, la littérature...).

5. Fonction structurante de l'organisation du cours : les récits servent à construire la "mémoire didactique ", à annoncer ce qui va suivre.

6. Fonction de support: le récit sert de support pour mettre en place, exposer, introduire des savoirs ou des savoir-faire (lire, écrire, parler, écouter, trier des informations, expanser, résumer, développer le sens de l'observation, l'imagination...). Le récit et sa construction ne sont pas importants en tant que tels, ce sont des prétextes (voir, par exemple, les classes de langues).

7. Fonction actualisante : le récit rend présent dans la classe ce qui ne peut y être présent pour de multiples raisons (passé, empan trop important, évènements « extérieurs », invisibilité6).

8. Fonction dynamisante: le récit «rend vivant» (voir les anecdotes, les témoignages, les histoires...). Cela s'articule avec une dimension implicative : le récit sert à engager l'élève dans le travail, à l'engager dans la communauté de travail (voir le « quoi de neuf? »). Il joue sur la relation entre cognition et affects (voir, par exemple, la relation aux personnages...).

9. Fonction de médiation cognitive : le récit sert de palier, de phase intermédiaire pour une étape, estimée intellectuellement supérieure par l'école (voir dans certaines Instructions officielles des cycles 1 et 2, le «langage d'évocation » qui est censé servir de propédeutique à l'entrée dans l'écrit ou encore les places respectives du récit, de l'explication et de l'argumentation dans la programmation des contenus au sein du cursus disciplinaire en français...).

10. Fonction normalisatrice ${ }^{7}$ : le récit construit une vision du monde, une vision de la discipline, des distinctions (fiction/réel, littéraire/non littéraire...), des valeurs (esthétiques, morales), des représentations des savoirs et de leur construction, des normes comportementales... Il vise ainsi à construire des sujets actifs, membres de communautés déterminées, engagés en fonction de ces normes et valeurs.

11. Fonction de mise en relation des disciplines: le récit construit des passerelles entre les disciplines, les mettant en relation et en interaction, les intégrant parfois (voir aussi bien Le Tour du monde par deux enfants que certaines expériences interdisciplinaires).

\section{Quels statuts dans le fonctionnement des disciplines?}

5 À partir de là, il me parait intéressant, dans une perspective de didactique comparée, de comparer les fonctionnements des récits dans les différentes disciplines, en prenant 
appui sur la typologie des statuts des contenus proposée par Y. Chevallard $(1991)^{8}$. Elle est en effet intéressante en ce qu'elle permet de comprendre les différentes strates de fonctionnement des disciplines. Ainsi, à partir des fonctions possibles, on peut s'apercevoir que dans certaines disciplines les récits ont un statut disciplinaire : ce sont des objets explicites d'enseignement, d'apprentissage et d'évaluation disciplinaires et ils peuvent, du coup, avoir un caractère plus ou moins emblématique de la discipline (c'est le cas en français, surtout en lecture-écriture, ainsi qu'en histoire dans certaines modalités de fonctionnement de cette discipline). Dans d'autres disciplines, les récits ont un statut paradisciplinaire: ils fonctionnent plutôt comme des outils, non enseignés explicitement, mais que les élèves doivent s'approprier en s'appuyant sur les répétitions et les pratiques du maitre. Ils peuvent encore avoir un statut protodisciplinaire: ce sont, en quelque sorte, des pré-requis, leur absence étant considérée comme un scandale ${ }^{9}$. Les récits ont, par voie de conséquence des modes de présence sémiotique différents (oral, écrit, pictural, mixte... ), des modes de fonctionnement différents dans les interactions en classe (réservés au maitre, aux élèves ou aux deux ; sollicités ou refusés...), des formes différentes (complets, lacunaires.......

\section{Quels effets sur les élèves ?}

6 Ces constats engendrent deux autres questions importantes dans un cadre didactique :

- Ces différences sont-elles expliquées aux élèves, par qui et comment?

- Comment les savoirs et les savoir-faire concernant les récits se construisent chez les élèves au travers des interactions entre fonctions et disciplines?

7 La première question implique de réfléchir aux travaux interdisciplinaires possibles; la seconde aux effets produits sur les élèves. Je dois dire que, sur ces questions, les travaux manquent ce qui constitue peut-être un indice complémentaire de la difficulté à construire des questions didactiques.

\section{Gestion des contenus et compétence narrative}

8 La question des contenus me semble importante si on admet que tout récit est récit de contenus et que le fait de pouvoir (se) dire directement ou indirectement contribue pour une bonne part à la motivation pour le travail d'écriture. De surcroit, J.-F. Halté (1981 ; 1984 ; 1987) avait montré au travers de la notion de malentendu communicationnel que la gestion des contenus constituait un lieu particulièrement problématique de l'écriture et de l'évaluation scolaires. En effet, la consigne demande un texte et laisse entendre que ce que l'élève peut écrire sur lui ou ce qu'il peut imaginer intéresse l'enseignant. Mais la réponse du maitre (les corrections) porte sur des unités plus réduites que le texte (phrases, mots...), essentiellement sur un mode orthographique et grammatical, c'est-àdire en tenant les contenus pour quantité négligeable.

9 C'est donc sur cette question des contenus, quelque peu délaissée par les didacticiens, que je souhaite revenir en m'appuyant notamment sur une recherche collective menée entre 2001 et 2006 (Reuter, 2007b) qui a pris comme objet un groupe scolaire (Concorde) d'une banlieue défavorisée de Lille (Mons-en-Barœul), dont tous les maitres appartenaient à l'ICEM (Institut coopératif de l'école moderne) ${ }^{10}$. 


\section{Choix des consignes et contenus mis en scène}

10 Deux consignes d'écriture sollicitant des récits aux contenus différents ont été données aux élèves à des fins évaluatives ${ }^{11}$. La première consigne était la suivante : «C'est le soir. Comme d'habitude un enfant se couche et lit, en attendant que son père vienne l'embrasser. Mais il tarde à venir. Soudain la porte s'ouvre. Une créature monstrueuse apparaît... ». Ce choix s'appuyait d'abord sur le fait que les récits sollicitant l'imagination (RSI) correspondent à une tradition scolaire importante en français et ont fait l'objet d'études quant à leurs réalisations et aux problèmes qu'ils soulèvent (Clanché, 1988, 1992 ; Kaïci, 1991, 1992). De surcroit, la consigne, avait été testée à de multiples reprises lors de travaux antérieurs de membres de notre équipe ou d'étudiants (Deschildt, 2004; Humbert-Prudhomme, 2004 et 2006 ; Lammertyn, 1999 et 2000). Je disposais donc de deux plans comparatifs. Elle présente, en outre, quelques spécificités intéressantes quant aux problèmes à traiter : ancrage dans le «réel » (voir les problèmes de catégorisation vérité/mensonge - des élèves), continuation au présent (qui s'oppose aux pratiques scolaires dominantes en matière de récits fictionnels, où l'usage du passé est presque contraint $t^{12}$ ), et absence de marquage du genre de l'enfant (ce qui permet d'étudier les projections possibles du narrateur).

11 La seconde consigne était la suivante : «Raconte une soirée que tu as réellement vécue et qui t'as marqué. » Outre le fait, qu'ici encore, j'avais expérimenté auparavant cette consigne auprès de nombreuses classes et disposais donc d'un arrière-plan comparatif substantiel, elle présentait à mes yeux plusieurs intérêts non négligeables: elle "marche» (i.e. les élèves écrivent); elle autorise une relation d'évènements (sans forcément passer par une mise en intrigue); elle sollicite le marquage émotionnel qui, d'un côté est lié à la mémorabilité des évènements mais de l'autre semble, au travers des études disponibles, un point faible commun à nombre d'écrits d'élève. De surcroit, les récits sollicitant le vécu (RSV) appartiennent à une catégorie d'écrits inscrite dans la tradition scolaire, mais avec des variations importantes dans les attendus et les implicites selon les époques et les types de pédagogie (Bishop, 2004, 2010); ils réclament des activités mentales au moins en partie différentes des RSI, puisqu'il s'agit de mettre en forme un référent pré-existant, plutôt que de construire un univers fictionnel et ils ont fait l'objet de multiples études (voir notamment Bishop, 2004; Clanché, 1988 et 1992; Fayol, 1984 et 1985 ; Labov, 1978 ; Lahire, 1993) mettant notamment au jour des problèmes tels que le rapport à la vérité, la sélection et la mémorisation des contenus, la mise en jeu de l'image de soi...

\section{Appréhender la compétence narrative?}

12 Cette recherche soulève une question qui me parait particulièrement importante dans un cadre didactique. En effet, la disparité des productions textuelles selon la consigne soulève le problème de l'analyse de ce que certains appellent la compétence narrative. On voit bien en effet que, selon ce qui est principalement sollicité (vécu ou imaginaire en l'occurrence), les élèves réalisent des écrits très différents sur plusieurs dimensions, quelles que soient les catégories d'élèves et la pédagogie pratiquée. Les élèves prennent ainsi plus de temps pour écrire les RSI qui sont plus longs et comprennent plus d'actions de premier plan. Ces récits manifestent aussi une plus grande diversité dans les moyens textuels employés, notamment en ce qui concerne le marquage de la pensée, les 
descriptions et les dialogues. Ils se caractérisent encore par des problèmes de gestion de la compréhension et des désignateurs plus importants ${ }^{13}$. Ils creusent aussi plus d'écarts entre les performances des élèves de différents milieux sociaux. Tout cela interroge la possibilité d'appréhender une éventuelle compétence narrative à partir d'une seule catégorie de récits.

13 Par voie de conséquence, il me semble difficile, en didactique, de se priver d'une diversification des consignes si l'on souhaite appréhender, de manière un tant soit peu précise, les compétences rédactionnelles des élèves dans ce domaine. Cela me parait en tout cas encourager la didactique à multiplier les recherches autour des effets des consignes (et des situations), de leurs intérêts et de leurs limites.

\section{Une analyse des récits prenant en compte les contenus?}

14 L'analyse des écrits des élèves pose en tout cas - ceci n'est pas nouveau (voir, par exemple, Fabre-Cols, 2000) - des problèmes redoutables, quant à la détermination des critères, à la construction des catégories et aux valeurs qui leur sont attribuées. Et, de fait, l'appréciation de la gestion des contenus par les élèves s'est avérée particulièrement complexe.

15 J'ai ainsi tenté d'analyser les contenus et leur mode de mise en scène non seulement sous l'angle de la langue et du texte mais aussi sous celui de l'imagination (et de l'investissement). La distinction entre ces deux dimensions (voire la nécessité ou la possibilité de le faire) constitue un vrai problème, peu thématisé dans les études antérieures sur le sujet ${ }^{14}$. Pour le pôle des contenus référant à l'imaginaire, j'ai pris en compte la « réalité » du monstre (à la différence d'autres scénarios tels l'illusion, la blague ou le rêve) ; les traits pouvant marquer la construction du scénario imaginaire (le nombre d'actions du monstre, l'affrontement, la fuite, l'action de se cacher, les risques pour la vie de l'enfant, le triomphe de l'enfant, l'issue négative, l'issue «superpositive $»^{15}$, l'amitié entre l'enfant et le monstre, le changement de genre entre l'auteur-élève et le narrateur) ; les traits susceptibles de marquer l'investissement; la transformation des parents en monstres ; les traces d'un « roman familial $»^{16}$, la victimisation de la famille; la solitude de l'enfant (lorsque ses cris ne sont pas entendus) ${ }^{17}$. Mais j'ai bien conscience que tout cela est discutable. Au moins j'aurais tenté de le faire et le pôle des contenus n'a pas disparu de l'horizon évaluatif qui était le mien dans cette recherche.

\section{L'intérêt d'un croisement des méthodes en didactiques}

16 Je souhaite maintenant expliquer ici en quoi en didactique(s) une approche cantonnée aux textes me parait insuffisante si on souhaite comprendre les difficultés auxquelles se heurtent les élèves et les problèmes qu'ils construisent (ou non). J'explorerai cela au travers de deux questions : celle de la vérité et celle de la rareté des dialogues.

\section{Vérité et statut didactique}

17 Les entretiens montrent que le problème principal que se posent les élèves lorsqu'ils écrivent un RSV réside dans le critère fondamental qui les guide, celui de la vérité: le texte doit dire le vrai, ce qui s'est réellement passé. Il s'agit, comme le disent certains, d'une «histoire qu'on ne peut changer » car, à la différence des écrits où l'on peut 
inventer, ici transformer serait mentir. On retrouve donc les conclusions classiques de P. Clanché (1992), confortées par celles de P. Lammertyn (2000) et de M.-F. Bishop (2004, 2010). Cela explique, entre autres, que ces textes soient ressentis comme fortement contraignants, à la différence de ceux qui sollicitent l'imaginaire. Cependant la rigidité de cette conception éthique de la vérité s'articule avec l'idée pragmatique d'une exhaustivité impossible ${ }^{18}$ : on peut oublier des choses; on ne peut pas tout dire (car ce serait trop long, le scripteur ou le lecteur se perdrait dans l'histoire...); on peut ne pas tout dire : il faut sélectionner en fonction d'une hiérarchisation de l'intérêt qui accorde la première place aux actions et secondarise paroles, descriptions, détails...

18 Cette conception, fondée sur la vérité, constitue la mémoire en ressort essentiel du travail rédactionnel. Il convient donc de chercher quelque chose dont on se souvienne bien (d'où l'intérêt d'évènements récents). Cela justifie une écriture rapide " pour ne pas oublier » et explique encore qu'une des fonctions principales des pauses dans l'écriture soit celle de la remémoration.

19 Les principales difficultés mentionnées par les élèves peuvent donc être regroupées autour de deux axes spécifiques ${ }^{19}$ : celui de la vérité (et de la mémoire) et celui de l'exposition du privé/personnel/intime. Mais cette catégorie du privé, telle qu'elle est construite par les élèves au travers de désignations variées ${ }^{20}$, est relativement complexe. Elle s'élabore autour de trois dimensions en interaction: la localisation (il s'agit essentiellement de ce qui se passe hors de l'école), le rapport à la personne (il s'agit de ce qui la «touche» vraiment: divorce des parents, décès d'un être cher, sentiment amoureux, paroles confiées...), le rapport à la communication (il s'agit de ce qui ne peut/ ne doit se dire qu'à soi-même $e^{21}$, en tout cas de ce qui ne peut se dire dans les circuits publics et scolaires ordinaires).

Les RSV ont donc un statut didactique problématique. Pour une majorité d'élèves, il est discutable de donner de telles consignes à l'école. Il n'existerait pas d'aides ou d'appuis possibles dans les films ou les autres écrits puisque les évènements et les sentiments ressentis sont uniques et singuliers. Pour les mêmes raisons, leur enseignement et leurs apprentissages seraient impossibles. En outre, ces textes seraient peu modifiables (sauf à la marge, sur l'orthographe par exemple) puisque le critère essentiel de pertinence est la conformité au réel que l'élève a mis en œuvre (et qu'il est le seul à pouvoir garantir). Que le maitre demande des modifications conséquentes est donc vécu comme difficilement acceptable.

21 Cette conception, sans doute discutable mais qui a le mérite de la cohérence, nécessite encore qu'on s'y arrête sur deux points qui confortent ce statut didactique problématique. En premier lieu, pour nombre d'élèves, l'appel au souvenir fait qu'on revit les évènements et les émotions, " on se remet dedans ", ce qui ne favorise sans doute pas une position distanciée quant au texte. En second lieu, et la remarque est récurrente, cela serait peu adapté aux enfants soit parce qu'ils ont moins de souvenirs que les adultes, soit parce qu'ils ont moins de choses intéressantes à dire que les adultes. L'opposition activée est, en tout état de cause, l'opposition adultes vs enfants.

\section{La rareté des dialogues}

22 Les entretiens permettent encore d'éclairer d'autres problèmes, telle la rareté des dialogues et des descriptions dans les RSV comparée à leur plus grande présence dans les RSI. 
Les élèves ont, ici encore, des positions convergentes quant à la présence du dialogue, quelle que soit la pédagogie dont ils relèvent. Cela est tributaire, pour eux, des principaux critères renvoyant au vécu, notamment souvenir et vérité (on a oublié les paroles exactes, il y avait trop de paroles, tout le monde parlait en même temps...) ainsi que privé et intime (c'est trop personnel, il s'agit de secrets confiés...). À ces critères s'ajoute celui de la hiérarchie: c'est secondaire par rapport aux actions, les paroles étaient peu importantes, les gens n'ont pas beaucoup parlé... On peut cependant remarquer, autour de ce noyau, d'autres critères mis en avant de manière plus disséminée : celui des normes scolaires (quand les paroles sont estimées trop vulgaires), celui de la longueur excessive (par exemple, l'oraison du père lors d'un enterrement) ou celui des problèmes de compréhension du maitre ou des pairs.

Les entretiens permettent ainsi de mieux comprendre l'absence relative de dialogues dans ces écrits en mettant au jour le socle commun d'appréhension sur lequel ils reposent. On voit donc que, si la visée est de préciser ce qui est de l'ordre des compétences, il est intéressant non seulement de diversifier les critères textuels et de comparer de manière conséquente (selon les catégories d'écrits et d'élèves) mais encore de mettre cela en relation avec des entretiens afin de ne pas attribuer de significations erronées à la présence ou à l'absence de tel phénomène ${ }^{22}$.

\section{De la position exotopique à la position d'apprentissage}

25 Je souhaite m'arrêter maintenant sur une question qui m'apparait de plus en plus importante : celle de la position d'apprentissage. Je repartirai pour ce faire de ce que certains appellent une position distanciée ou une position exotopique ${ }^{23}$.

\section{Interroger la position exotopique}

Pour interroger cette catégorie de "position exotopique», je m'appuierai, trop brièvement sans doute, sur le texte de Louis $^{24}$, en réponse à la question sollicitant l'imaginaire.

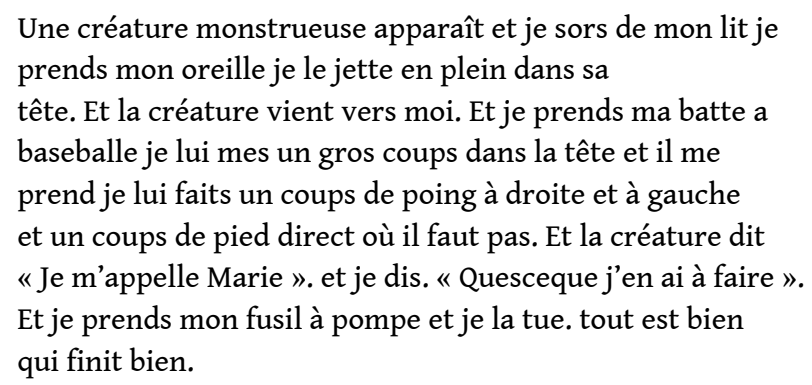

Une analyse rapide pourrait conclure à une position peu distanciée (ou non exotopique) mais l'entretien mené révèle une véritable réflexion sur plusieurs points : le problème du démarrage dans la mesure où Louis s'interroge sur l'endroit où peut se trouver le père, une volonté d'humour (faire de Marie, une élève qu'il n'aime pas, le monstre), une réflexion sur les phrases du dialogue qui doivent faire comprendre les relations entre les personnages et éviter les injures en raison des normes scolaires, la recherche d'une fin cohérente... Cela montre à mon sens, que la détermination d'une quelconque position (ou posture) ne peut être déterminée à partir du texte seul ${ }^{25}$. 


\section{La position d'apprentissage} amélioration possible du texte.

-Louis. Les copains ils comprennent mieux que... monsieur et les adultes. Et ben ça marche mieux parce qu'on connaît des choses que les adultes ne savent pas. Et mon humour fonctionne bien avec eux alors qu'avec les adultes c'est pas ça.

- E. Donc tu aurais décrit ton monstre si ton texte était destiné à tes copains parce qu'avec eux c'est possible, ils comprennent ton humour alors que monsieur et les adultes non?

- Louis. Ouais avec des enfants ça passe mieux.

- E. Ça passe mieux avec des enfants qu'avec des adultes?

- Louis. Ben oui, ils sont stricts les adultes, ben sois poli, va vite dans ta chambre, ne dis pas du mal... euh... ben plein de trucs comme ça.

- E. Et au niveau de ton texte?

- Louis. Ben je pouvais pas écrire d'insultes... déjà... du sang... plus d'horreur.

- E. Donc tu n'as pas osé faire un texte avec des personnages qui s'insultent et où il $\mathrm{y}$ a du sang et des choses horribles parce qu'il va être lu par des adultes, c'est ça ? -Louis. Ben oui c'est monsieur T. qui corrige et..

Cette même position se retrouve aussi dans l'entretien mené avec Jérémy.

- E. Et si monsieur te demande de modifier ton histoire? Qu'il te dise, ton histoire me plaît bien, mais j'aimerais que tu la modifies un peu ça?

- J. Ben ça, monsieur ne nous le dit jamais.

- E. Oui, mais supposons qu'il te demande, qu'en penses-tu?

- J. Ben non, je l'ai fait comme ça.

- E. Tu l'as fait comme ça?

-J. Ben je l'ai imaginé, c'est mon histoire.

-E. Donc tu veux bien corriger tes fautes de grammaire mais tu veux pas modifier ton histoire parce que toi tu l'as imaginée comme ça?

-E. Oui.

Dans le cas de Marie, si le maitre est toujours perçu en tant qu'adulte, il n'y a, en revanche pas de contestation sous une forme ou une autre: on doit se soumettre, c'est une évidence.

- E. Que penses-tu de ces corrections?

- M. C'est normal, c'est un maître, nous on est des enfants, on doit suivre le chemin des plus grands, plutôt que de faire notre chemin, et se planter dans un mur.

- E. Tu es toujours d'accord avec les corrections du maître?

- M. Ben oui.

Les élèves qui travaillent dans le cadre de la pédagogie Freinet témoignent en revanche d'une position tendanciellement différente ${ }^{28}$ que je qualifierais d'apprentissage ou de plus 
autonome. Ainsi, même dans le cas des RSV, dont a vu précédemment le statut didactique problématique, on trouve chez eux des explications et des justifications plus précises accompagnées d'exemples concernant le choix des idées, les procédures de mémorisation, ce qui est important ou secondaire. Mais ce qui diffère fondamentalement réside dans le rapport aux normes scolaires et à l'autorité du maitre. Ainsi des élèves expliquent qu'ils n'ont pas respecté le cadre temporel (la soirée) imposé par la consigne dans la mesure où le souvenir qu'ils avaient sélectionné leur semblait plus marquant ${ }^{29}$. En outre, l'autorité du maitre n'est pas envisagée comme un pouvoir arbitraire et/ou absolu et le maitre luimême est considéré comme un partenaire avec qui il est possible de discuter. La position évaluative, telle qu'elle se manifeste au travers de ces déclarations, est aussi différente : plus complexe et plus nuancée. Les critères invoqués, par exemple, portent souvent sur un plus grand nombre de dimensions (normes scolaires, textuelles, génériques...). On rencontre aussi, moins qu'ailleurs, des discours affichant une satisfaction absolue quant à la réussite du texte. En revanche, plus qu'ailleurs, on rencontre soit un sentiment de réussite relatif et argumenté ou mitigé avec l'indication explicite de ce qui est perçu comme moins réussi et de ce qu'il aurait fallu faire, soit un sentiment de "ratage », lui aussi explicité. Dans tous les cas, cette évaluation est référée à la consigne et au projet textuel communicationnel ${ }^{30}$. La dimension communicationnelle s'avère fondamentale tendant à prendre le pas sur les autres ${ }^{31}$. Elle s'appuie constamment, sur des dispositifs précis et diversifiés. Ceux-ci peuvent concerner les interactions individuelles avec le maitre ressenties sur le mode de la négociation et non réduites à des renvois sur l'orthographe, la conjugaison ou la syntaxe, mais comportant aussi des questions (pour mieux comprendre) et des suggestions quant à la lisibilité, aux moyens textuels à utiliser, à la cohérence («ce qui ne suit pas dans le texte»), aux informations nécessaires... Ces dispositifs concernent encore les interactions avec les autres élèves, considérés le plus souvent comme un collectif pouvant intégrer le maitre ${ }^{32}$. Cela explique qu'on n'ait quasiment jamais de relation unique aux «copains » et jamais d'opposition adulte vs enfant comme ailleurs. Ces dispositifs sont, par ailleurs, restitués dans leur diversité générique (textes libres, entretiens, correspondances...) et communicationnelle (on peut écrire et montrer ou non ses textes aux autres, écrire et les mettre ou non dans les instruments disponibles: brochures, cahier de classe...), cette dernière dimension confortant la réflexion sur les choix possibles. Resituées dans ce cadre, les questions du privé et de l'intime ainsi que du statut didactique des textes sollicitant le vécu se posent différemment. Ainsi, pour ces élèves, une telle demande est possible - même si elle est loin d'être évidente - puisque, par exemple, certains élèves se disent déjà (dans les entretiens) ou écrivent déjà (dans les textes libres) ce genre de choses, puisque certains peuvent déjà l'écrire sans le lire à tous ou sans l'écrire dans le cahier de classe, et puisqu'ils peuvent même refuser cette demande. Complémentairement, il est possible de modifier, sur certains points, le texte sans mentir, notamment pour que les autres comprennent mieux, ce qui ouvre un espace d'acceptabilité pour les interventions du maitre : «enlever le moins important», changer les choses «trop rapides» ou «trop longues "... pourvu qu'elles permettent d'«arranger le texte» en le rendant plus intéressant pour le lecteur. Du coup, pour certains élèves, des apprentissages liés à ce genre d'écrits deviennent possibles. 


\section{Position d'apprentissage, statut didactique et relation aux configurations disciplinaires}

$34 \mathrm{Au}$ travers de ces analyses je rejoins certains des résultats d'études antérieures, notamment les conclusions de M.-F. Bishop (2004, p. 449-464) qui montre les problèmes que soulèvent les «écritures de soi scolaires " par rapport au contrat didactique, aux autobiographies littéraires et aux écritures ordinaires. Nombre de ces problèmes sont effectivement partagés par l'ensemble des élèves, quelle que soit la pédagogie dont ils relèvent. De fait les écrits sollicitant le vécu semblent tendanciellement être ressentis comme une intrusion dans la vie privée, et être difficilement évaluables et modifiables et peu susceptibles d'apprentissages. On peut donc poser que leur statut didactique est à priori problématique.

Il n'en reste pas moins vrai, qu'au-delà de ce consensus, des différences ont pu être constatées dans les écrits eux-mêmes et dans l'appréhension de la tâche. Pour le dire vite, dans le cadre de la pédagogie "Freinet ", ce statut semble moins problématique pour les élèves, puisque ce genre d'écrits est appréhendé au travers d'une configuration disciplinaire différente.

Je désigne par cette expression de "configuration disciplinaire » (Reuter \& LahanierReuter, 2007; Reuter, 2011; 2014a ; 2014b) le fait qu'une discipline s'actualise sous des formes très différentes selon les époques, les pays, les moments du cursus, les filières, les pédagogies... Et, de fait, la configuration disciplinaire du français est différente dans le cadre de la pédagogie "Freinet» (Giguère, 2005; Reuter, 2006 et 2009) et la consigne portant sur des RSV est appréhendée au travers de dispositifs communicationnels et évaluatifs et d'une diversité de pratiques de production et de réception qui rendent ces textes moins attentatoires à l'espace intime, plus acceptables, plus susceptibles de modification... Le statut didactique de ce genre d'écrits varie donc selon les pédagogies.

L'ensemble de ces remarques génère à mon avis quelques conséquences quant aux recherches en didactique. En premier lieu, il me semble important de prendre en compte la position d'apprentissage bien plus que les positions dites distanciées quant aux textes ou quant au rapport au langage ${ }^{33}$. Cela parce que les interventions enseignantes produisent des configurations disciplinaires ${ }^{34}$ et peuvent donc générer des positions plus moins propices aux apprentissages et transformer les représentations, les rapports et les fonctionnements initiaux. La seconde conséquence me parait résider dans la nécessité de développer des recherches afin d'affiner nos connaissances sur les variations des performances textuelles et de la position d'apprentissage selon les catégories d'écrits sollicités, et selon la configuration disciplinaire mise en place. La troisième conséquence que je mentionnerai ici ${ }^{35}$ serait de mieux explorer l'articulation entre contenus, modes d'enseignement et modes d'apprentissage ou, sous une autre forme, entre didactique et pédagogie. La dernière conséquence porte sur la plus extrême prudence quant aux propositions d'intervention dans les classes que ces recherches pourraient, même à leur corps défendant, laisser accréditer. Cela non seulement en raison des résultats lacunaires et parfois difficilement interprétables dont on dispose mais aussi parce qu'à partir d'un statut à priori problématique peuvent se construire des choix d'enseignement différents : abandon, utilisation d'autant plus forte que de tels écrits posent des problèmes importants aux élèves, changement de configuration disciplinaire... 


\section{Un dernier texte sur le chemin de la didactique...}

38

$$
\begin{aligned}
& \text { C'est le soir ou mes parents on dit que } \\
& \text { le père Noël n'existé pas. Mes parents } \\
& \text { quand ils ont dit ça j'allait donner } \\
& \text { un verre de lait au père Noël et } \\
& \text { une carote a c'est rène. }
\end{aligned}
$$

Je ne voudrais pas conclure cet article sans soumettre aux lecteurs un texte qui m'a beaucoup fait réfléchir depuis 2001. Il a été écrit par un garçon de CM2, venant d'un milieu défavorisé dans une école pratiquant une pédagogie classique. La consigne était «Racontez une soirée que vous avez réellement vécue et qui vous a marquée ».

Ce qui m'intéresse ici tient en cinq points :

- il s'agit d'un récit pour cet élève et, selon le cadre théorique qu'on sollicite sur la narrativité, on pourra ou non le considérer comme tel ;

- on peut considérer qu'il met en scène une émotion forte ressentie par cet élève (celle-ci faisant écho ou non à des ressentis chez le maitre) ;

- on peut considérer que son caractère laconique donne au texte une sobriété et une dignité qui contribuent à sa force ;

- on peut aussi considérer qu'il est améliorable, soit sur la dimension des normes scolaires du récit, soit sur la dimension de la mise en scène des émotions (soit sur ces deux plans);

- on peut considérer soit qu'il faut intervenir, soit juste entendre et apprécier (afin notamment de ne pas désapproprier l'élève de son texte et de ses émotions).

Il s'agit là encore, me semble-t-il, de questions propres aux didactiques qui ouvrent à des recherches à mener et que nulle autre discipline ne mènera à leur place...

\section{BIBLIOGRAPHIE}

ASTOLFI, J.-P. (2008). La Saveur des savoirs. Disciplines et plaisir d'apprendre. Paris : ESF.

AUDIGIER, F. (2007). « De l'usage de récits pour dire l'histoire, la géographie, l'éducation citoyenne ». Pratiques 133-134, p. 77-99. En ligne : https://www.persee.fr/doc/ prati_0338-2389_2007_num_133_1_2139.

BAKHTINE, M. (1984) [1979]. Esthétique de la création verbale. Trad. du russe par A. Aucouturier. Paris : Gallimard.

BISHOP, M.-F. (2004). Les Écritures de soi à l'école primaire de 1850 à 2004. Places, fonctions et enjeux. Thèse en sciences de l'éducation : Université Charles-de-Gaulle - Lille 3.

BISHOP, M.-F. (2010). « Racontez vos vacances... ». Histoire des écritures de soi à l'école primaire (1882-2002). Grenoble : Presses universitaires de Grenoble.

BRUNER, J. (1991) [1990]. Car la culture donne forme à l'esprit. De la révolution cognitive à la psychologie culturelle. Trad. de l'anglais par Y. Bonin. Paris : Eshel. 
BRUNER, J. (2000) [1986]. Culture et modes de pensée. L'esprit humain dans ses œuvres. Trad. de l'anglais par Y. Bonin. Paris : Retz.

CHEVALLARD, Y. (1991) [1985]. La Transposition didactique. Du savoir savant au savoir enseigné. Grenoble : Éditions La Pensée Sauvage.

CLANCHÉ, P. (1988). L'Enfant écrivain. Génétique et symbolique du texte libre. Paris : Éditions Le Centurion.

CLANCHÉ, P. (1992). « L'enfant de neuf ans, le réel et l'imaginaire ». Cahiers Binet-Simon 632, p. 21-35.

COHEN-AZRIA, C. (2007). «Les récits en sciences à l'école : première approche ». Pratiques 133-134, p. 125-141. En ligne : https://www.persee.fr/doc/prati_0338-2389_2007_num_133_1_2141.

DAUNAY, B. \& DENIZOT, N. (2007). « Le récit, objet disciplinaire en français ? ». Pratiques 133-134, p. 13-32. En ligne : https://www.persee.fr/doc/prati_0338-2389_2007_num_133_1_2136.

DELEPLACE, M. (2007). « Le récit comme accès à la connaissance historique. Réflexions didactiques sur le récit historique ». Pratiques 133-134, p. 33-53. En ligne : https://www.persee.fr/doc/ prati_0338-2389_2007_num_133_1_2137.

DESCHILDT, S. (2004). L'Écriture de récits sollicitant l'imaginaire au CM2. Mémoire de DEA en sciences de l'éducation : Université Charles-de-Gaulle - Lille 3.

FABRE-COLS, C. (dir.) (2000). Apprendre à lire des textes d'enfants. Bruxelles : De Boeck-Duculot.

FAYOL, M. (1984). « La distanciation dans le langage : l'exemple du calcul de l'origine dans le récit écrit d'expérience personnelle chez l'enfant de 6 à 10 ans ». Enfance 1, p. 5-19. En ligne : https:// www.persee.fr/doc/enfan_0013-7545_1984_num_37_1_2827.

FAYOL, M. (1985). Le Récit et sa construction. Une approche de psychologie cognitive. Neuchâtel : Delachaux et Niestlé.

GIGUÈRE, J. (2005). «L'univers de l'écrit à l'école primaire : comparaison de trois modes de travail pédagogique (pédagogie Freinet, pédagogie par projet, pédagogie “classique") ». In : Reuter, Y. (dir), Démarches pédagogiques et lutte contre l'échec scolaire (2002-2005). Rapport de recherche de l'ERTe 1021, t. 1, p. 177-211.

HALTÉ, J.-F. (1981). «Pour changer l'écrire ». Pratiques 29, p. 23-46. En ligne : https:// www.persee.fr/doc/prati_0338-2389_1981_num_29_1_1189.

HALTÉ, J.-F. (1984). « L'annotation des copies, variété ou base du dialogue pédagogique ». Pratiques 44, p. 61-69. En ligne : https://www.persee.fr/doc/prati_0338-2389_1984_num_44_1_2463.

HALTÉ, J.-F. (1987). « Les conditions de production de l'écrit scolaire ». In : Chiss, J.-L. et al. (éds), Apprendre/enseigner à produire des textes écrits. Bruxelles : De Boeck, p. 35-44.

HUMBERT-PRUDHOMME, C. (2004). Écrire des récits imaginaires au CM2. Analyse de productions et d'entretiens. Mémoire de maitrise en sciences de l'éducation : Université Charles-de-Gaulle - Lille 3.

HUMBERT-PRUdHOMme, C. (2006). Les Récits sollicitant le vécu. Analyse de productions d'élèves au CM2. Mémoire de master 2 en sciences de l'éducation : Université Charles-de-Gaulle - Lille 3.

KAÏCI, A. (1991). La Pratique de la rédaction au cycle d'observation des collèges. Analyse des effets de quelques modes de sollicitation à l'écriture appliqués à des élèves en échec. Thèse en sciences de l'éducation : Université de Bordeaux 2. 
KAї̈I, A. (1992). « L'histoire d'un enfant perdu ». Cahiers Binet-Simon 632, p. 9-19.

LABOV, W. (1978) [1972]. Le Parler ordinaire. Trad. de l'américain par A. Kihm. Paris : Éditions de Minuit.

LAHANIER-REUTER, D. (2007). « Récit dans la classe de mathématiques ». Pratiques 133-134, p. 101-123. En ligne : https://www.persee.fr/doc/prati_0338-2389_2007_num_133_1_2140.

LAHIRE, B. (1993). Culture écrite et inégalités scolaires. Lyon : Presses universitaires de Lyon.

LAMMERTYN, P. (1999). Écrire un récit imaginaire au CM2. Analyse des difficultés et des stratégies des élèves. Mémoire de maitrise : Université Charles-de-Gaulle - Lille 3.

LAMMERTYN, P. (2000). « La sollicitation de l'imaginaire dans l'écriture des récits : intérêts et problèmes ». Repères 21, p. 53-77. En ligne : https://www.persee.fr/doc/ reper_1157-1330_2000_num_21_1_2327.

PATRON, S. (dir.) (2018). Introduction à la narratologie postclassique. Les nouvelles directions de la recherche sur le récit. Villeneuve d'Ascq : Presses universitaires du Septentrion.

PIER, J. (dir.) (2007). Théorie du récit. L'apport de la recherche allemande. Trad. de l'allemand par T. Gallèpe et al. Villeneuve d'Ascq : Presses universitaires du Septentrion.

REUTER, Y. (2006). « Les récits sollicitant le vécu au CM2. Éléments d'analyse et de comparaison ». Repères 34, p. 131-139. En ligne : https://www.persee.fr/doc/

reper_1157-1330_2006_num_34_1_2732.

REUTER, Y. (2007a). « Récits et disciplines scolaires. Présentation du numéro ». Pratiques 133-134, p. 3-12. En ligne : https://www.persee.fr/doc/prati_0338-2389_2007_num_133_1_2134.

REUTER, Y. (2009). «Les récits de fiction en dernière année de primaire. Éléments d'analyse et de comparaison selon les pédagogies ». In : Dufays, J.-L. \& Plane, S. (éds), L'Écriture de fiction en classe de français. Namur : Presses universitaires de Namur, p. 69-85.

REUTER, Y. (2011). «Penser la perspective didactique : la question de l'articulation entre disciplinaire, pédagogique et scolaire ». In : Daunay, B., Reuter, Y. \& Schneuwly, B. (dirs), Les Concepts et les méthodes en didactique du français. Namur : Presses universitaires de Namur, p. 35-60. REUTER, Y. (2014a). « Didactiques et disciplines : une relation structurelle ». Éducation et didactiqu e 8 (7), p. 53-64. En ligne : https://journals.openedition.org/educationdidactique/1877.

REUTER, Y. (2014b). « Construire la catégorie de discipline scolaire en didactique(s) ». Linguarum Arena 5, p. 79-95.

REUTER, Y. (2016). « Questions aux didactiques ». In : Petitjean, A. (dir.), Didactiques du français et de la littérature. Metz : Crem, Université de Lorraine, p. 97-115.

REUTER, Y. (dir.) (2007b). Une école Freinet. Fonctionnements et effets d'une pédagogie alternative en milieu populaire. Paris : L'Harmattan.

REUTER, Y. (dir.) (2013) [2007]. Dictionnaire des concepts fondamentaux des didactiques. Louvain-laNeuve : De Boeck.

REUTER, Y. \& LAHANIER-REUTER, D. (2007) [2004]. « L'analyse de la discipline : quelques problèmes pour la recherche en didactique ». In : Falardeau, E. et al. (dirs), La Didactique du français. Les voies actuelles de la recherche. Québec : Presses de l'université Laval, p. 27-42. 
RUELLAN, F. (2000). Un mode de travail didactique pour l'enseignement-apprentissage de l'écriture au cycle 3 de l'enseignement primaire. Thèse en sciences de l'éducation : Université Charles de Gaulle Lille III.

VIEL, A. \& GIRAUlt, Y. (2007). « Nature mise en récits ». Pratiques 133-134, p. 143-163. En ligne :

https://www.persee.fr/doc/prati_0338-2389_2007_num_133_1_2142.

\section{NOTES}

1. De surcroit à dimension applicationniste.

2. C'est tout l'objet du Dictionnaire des concepts fondamentaux des didactiques et d'un certain nombre d'articles que j'ai pu écrire sur le sujet (Reuter, 2014a ; 2014b ; 2016).

3. Voir, par exemple, Pier (2007) ou Patron (2018).

4. Typologie discutable comme toute typologie mais dont la valeur heuristique m'intéresse pour les questions que je traite ici. Voir aussi, dans le même numéro ( Pratiques, 2007), les contributions de F. Audigier, C. Cohen-Azria, M. Deleplace, B. Daunay \& N. Denizot, D. Lahanier-Reuter, A. Viel \& Y. Girault...

5. Voir, par exemple, le cas de la maternelle.

6. Voir ce qui se passe dans la pensée des acteurs ( $c f$. les narrations de recherche).

7. Voir les travaux de J. Bruner $(1991 ; 2000)$ sur ces questions.

8. Je procède à quelques reformulations.

9. Par exemple, ne pas savoir lire au lycée.

10. Ce mouvement regroupe les enseignants se réclamant peu ou prou de la pédagogie Freinet.

11. Il s'agissait en effet de tenter d'appréhender les effets de la "pédagogie Freinet ». Dans ce cadre, j'ai comparé les récits produits par les élèves de cette école avec ceux d'élèves d'autres écoles travaillant selon d'autres pédagogies (voir sur les récits sollicitant le vécu, Reuter, 2006, et sur ceux sollicitant l'imagination, Reuter, 2009).

12. Au moins au primaire en France.

13. Ce qui est à mettre en relation avec la longueur des textes.

14. Cette remarque me concerne aussi. J'ai de surcroit tenté de penser l'équilibre ou le déséquilibre entre la dimension langagière et la dimension des contenus.

15. Caractérisée notamment par une mise en scène hyperbolique du bonheur final: organisation d'une grande fête, affirmation qu'on sera toujours heureux...

16. Parents éliminés au profit de l'amitié monstre-enfant, meurtre du monstre devenu l'ami de l'enfant par le père, partage du lit du monstre ou des parents par l'enfant, meurtre du père par le monstre qui partage le lit de la mère, actions référables à de la pédophilie...

17. J'ai synthétisé dans un indicateur complémentaire le nombre de traits présents renvoyant, selon moi, à l'imaginaire et à l'investissement, leur diversification étant, ici encore, considérée comme une marque de « réussite ».

18. Il revient à M.-F. Bishop d'avoir mis au jour cela. 
19. Outre un axe non spécifique à cette catégorie d'écrits, celui des normes langagières : orthographe, syntaxe...

20. « Secrets », « secrets de famille » ...

21. Via des écrits « pour soi » ou à des alter-ego, par exemple le club de lecture d'écrits intimes de quatre amies.

22. Il n'en reste pas moins que l'usage des entretiens est parfois quelque peu «fasciné » en didactique en oubliant qu'ils font véritablement sens dans une comparaison, relativement importante, selon les tâches et les élèves, afin de saisir les éventuelles spécificités de ce qui se dit. Ils soulèvent aussi des problèmes très concrets de faisabilité et de pertinence si on les sollicite en relation avec des productions. En effet, soit on les réalise très rapidement après celles-ci, avec un gain quant à la mémorisation de la tâche chez les élèves mais une perte quant à l'analyse préalable des écrits par le chercheur, soit on les réalise plus tardivement avec des intérêts et des limites inverses.

23. Voir B. Lahire (1993) qui s'appuie sur les travaux de M. Bakhtine (1984).

24. Issu des travaux de C. Humbert-Prudhomme. J'ai respecté la disposition ainsi que l'orthographe du texte.

25. L'espace me manque dans cet article pour montrer que ces positions (ou postures) sont bien loin d'être monolithiques, aussi bien dans les textes que dans les entretiens.

26. Cette dichotomie est trop tranchée : il s'agit plutôt d'un continuum.

27. Je m'appuie, ici encore, sur les entretiens menés par C. Humbert-Prudhomme.

28. Cela se manifeste déjà dans les interactions avec les chercheurs, ce dont rend compte la longueur, en général plus importante, des entretiens et de leurs interventions : ils parlent et s'affirment plus, allant parfois (ce qui est extrêmement rare chez d'autres élèves) jusqu'à exprimer leur désaccord, indiquer explicitement qu'ils n'ont pas compris la question ou même demander de la reformuler.

29. Cela amène donc à relativiser la valeur attribuée au respect de la consigne puisque, dans ce cas, il ne s'agit pas d'une absence d'attention ou de contrôle mais d'une véritable réflexion appuyée sur une autonomie dans l'accomplissement de la tâche.

30. Et non, simplement, à ses sentiments ou à l'effet provoqué (le rire par exemple) chez ses copains, comme c'est souvent le cas dans d'autres classes.

31. Il est ainsi symptomatique de remarquer que c'est uniquement chez ces élèves qu'on prête aux chercheurs ayant sollicité ces écrits des intérêts autres que langagiers ou textuels ( "mieux nous connaître ») et qu'on cherche réellement à les intéresser ( faire partager notre plaisir », « il faut que ce soit une soirée extraordinaire pour intéresser les chercheurs »). Les chercheurs sont ainsi constitués en partenaires de la communication.

32. Le « collectif-classe » débat pour aider l'auteur lors des entretiens, des textes libres, des recherches...

33. Surtout si elles sont envisagées de manière uniforme et sans appui sur des entretiens...

34. Il importe donc aussi de préciser ce qui, dans certaines configurations disciplinaires, fait obstacle et, dans d'autres, "Freinet» ou pas, aide à constituer des positions d'apprentissage.

35. Ce sera d'ailleurs pour moi l'occasion de rendre hommage à deux chercheurs, J.-

F. Halté et F. Ruellan (2000) qui ont constamment porté l'accent là-dessus. 


\section{RÉSUMÉS}

Dans cet article, après avoir rappelé le cadre didactique "autonomiste » au sein duquel je me situe, je reviens sur plusieurs recherches menées sur le récit afin de proposer quelques pistes de réflexion sur des questions proprement didactiques. Ces questions concernent notamment les fonctions des récits dans les différentes disciplines, la prise en compte des contenus dans l'analyse des textes d'élèves, les méthodes d'analyse pertinentes en didactique, le statut didactique des types de récits et la position d'apprentissage.

In this paper, I first recall the "autonomist" didactical framework I use. Afterwards I put a new glance on some researches upon narrative, and present some reflexion that deal with strictly didactical issues. These issues are: the narratives' functions in different school-subjects, the consideration didactics must have towards the contents of pupils' texts, the appropriate analyse methods didactics have to develop, the didactical status of narratives and the learning position.

\section{INDEX}

Mots-clés : récit, didactiques, statut disciplinaire des récits, position d'apprentissage, fonctions des récits

Keywords : narrative, didactics, narrative disciplinary status, learning position, narrative functions

\section{AUTEUR}

\section{YVES REUTER}

Université de Lille, Théodile-CIREL, F-4900 Lille, France 